Please do not remove this page

RMIT

UNIVERSITY

\title{
The rheological behaviour of anaerobic digested sludge
}

Baudez, J; Markis, Flora; Eshtiaghi, Nicky; Slatter, Paul

https://researchrepository.rmit.edu.au/esploro/outputs/9921858353101341/filesAndLinks?institution=61RMIT_INST\&index=null

Baudez, J., Markis, F., Eshtiaghi, N., \& Slatter, P. (2011). The rheological behaviour of anaerobic digested sludge. Water Research, 45(17), 5675-5680. https://doi.org/10.1016/j.watres.2011.08.035

Document Version: Submitted Version

Published Version: https://doi.org/10.1016/j.watres.2011.08.035

Repository homepage: https://researchrepository.rmit.edu.au

(C) 2011 Elsevier Ltd. All rights reserved

Downloaded On 2023/04/27 00:27:19 +1000

Please do not remove this page 
Citation:
Baudez, J, Markis, F, Eshtiaghi, N and Slatter, P 2011, 'The rheological behaviour of anaerobic digested sludge', Water Research, vol. 45 , no. 17 , pp. $5675-5680$.

The anaerobic rheological behaviour of digested sludge

J.C. Baudez ${ }^{1.2}$, F. Markis ${ }^{2}$, N., Eshtiaghi², P. Slatter ${ }^{2}$

1 Cemagref, UR TSCF, F-03150 Montoldre, France

2 Rheology and Materials Processing Centre, Dept. of Chemical Engineering, RMIT University, Victoria, Australia, 3001

Corresponding author: jean-christophe.baudez@cemagref.fr

Abstract

Producing biogas energy from the anaerobic digestion of wastewater sludge is one of the most challenging tasks facing engineers, because they are dealing with vast quantities of fundamentally scientifically poorly understood and unpredictable materials; while digesters need constant flow properties to operate efficiently. An accurate estimate of sludge rheological properties is required for the design and efficient operation of digestion, including mixing and pumping. In this paper, we have determined the rheological behaviour of digested sludge at different concentrations, and highlighted common features. At low shear stress, digested sludge behaves as a linear viscoelastic solid, but shear banding can occur and modify the apparent behaviour. At very high shear stress, the behaviour fits well to the Bingham model. Finally, we show that the rheological behaviour of digested sludge is qualitatively the same at different solids concentrations, and depends only on the yield stress and Bingham viscosity, both parameters being closely linked to the solids concentration.

Keywords

Digested sludge, Bingham model, Herschel-Bulkley model, shear banding, viscoelasticity.

Introduction

Renewable energy is said to be one of the pillars of sustainable management. Biogas from the anaerobic digestion of sewage sludge can provide a clean, easily controlled source of renewable energy from sewage sludge, replacing fossil fuels. However, an accurate estimate of sludge rheological properties is required for the design and efficient operation of the pumping systems which surround anaerobic digesters (Slatter, 1997, 2003). Indeed Tarp and Melbinger (1967) showed the significant advantages of recycling and recirculating digested sludge to mix it with 
excess sludge, among them an increase in biogas production (Sperry, 1959). The mixture can be concentrated to a much higher solid content than would be possible for the excess sludge alone, and recirculation also facilitates improved mixing efficiency over mechanical stirring. However, the flow rate in the recirculation circuits has to be very large (Appels et al, 2008) and rheology is needed to calculate head losses and pumping power (Slatter, 2001).

Except for the work of Monteiro (1997) who showed that anaerobic digestion induces a decrease of the rheological characteristics of sludge, most investigations on sludge rheology were focused on activated sludge. No reliable data, at high shear rate (within recirculation pipes), can be found in the literature for digested sludge while at low shear rate (within the digester), results are scarce and not always usable. Most of these were obtained by applying shear rate ramps that gave distinct peaks in the flow curve (for example, Ayol et al., 2005), but Baudez (2006) clearly established that these peaks in the flow curves were principally instrument artefacts, and not material characteristics. However, the work of Ayol et al. (2005) pointed out that with very dilute sludge, the Ostwald model, i.e. a power law model with no yield stress, gave the best fit.

From a physical perspective, digested sludge appears to be a stable suspension with low settling rates (Namer and Ganczarczyk, 1993) and low surface charge (Forster, 2002), implying that interactions are more steric than electrostatic. The most important constituents in digested sludge are lipopolysaccharides (Forster, 1983) which are amphiphile lipids with both hydrophilic and hydrophobic heads. These molecules displayed a very intriguing rheological behaviour (Muñoz et al, 2000), showing linear viscoelasticy, non-Newtonian viscous flow and shear banding (Miller and Rothstein, 2007).

In this paper, our intention is to establish the basic characteristics of the rheological behaviour of digested sludge, with the objective of industrial applications in digester mixing, pumping and pipe flows, meaning that we will focus on short-term behaviour. Short-term behaviour means we will not focus our research on eventual thixotropic effects. As predicted by the literature on amphiphile rheology, we show that digested sludge exhibits linear viscoelastic behaviour at low shear stresses, followed by shear-banding phenomena at intermediate stresses, and finally a nonNewtonian fluid behaviour with a yield stress, modelled by a Herschel-Bulkley model at intermediate shear rates and by a Bingham model at very high shear rates. We also highlight the fact that the rheological behaviour is qualitatively the same at different solid concentrations, allowing us to define a master-curve for which the dimensionless parameters are the yield stress

Material and methods

The digested sludge was sampled at the Mount Martha waste water treatment plant (Melbourne, Victoria, Australia) at the outlet of the digester number 1. Its initial solid concentration was at 18.5g. $\mathrm{L}^{-1}$ and was also gently concentrated to $25.5,32$ and $49 \mathrm{~g} \cdot \mathrm{L}^{-1}$ by using a Buchner vacuum. Sludge samples were stored at $4^{\circ} \mathrm{C}$ for 30 days befor e experiments, in order to reduce temporal variability. Indeed, even after anaerobic digestion, sludge may not be fully stabilised and organic 
changes may still occur. By storing the sludge sample for such an extended period, the potential for composition changes is reduced; and we can assume that we used exactly the same material throughout all our experiments.

Rheological measurements were performed with a DSR200 instrument from Rheometric Scientific, connected to a temperature controlled water bath. The rheometer was equipped with a cup and bob geometry (inner diameter: $29 \mathrm{~mm}$, outer diameter: $32 \mathrm{~mm}$, length: $44 \mathrm{~mm}$ ). Temperature was kept at ${ }^{\circ} 5^{\circ}$. To avoid evaporation, sludge was cove red with a thin film of immiscible Newtonian oil.

Before each measurement, sludge was presheared for 10 minutes at a shear rate of $1000 \mathrm{~s}^{-1}$ then left at rest for 10 minutes. This procedure allowed us to erase material memory and to have reproducible measurements. Then, different tests were performed:

- Shear stress sweep, by applying a linear ramp of increasing stress over time. In this test, we changed the time of rest between preshear and shear, from 1 to 60 minutes in order to investigate structural changes occurring during rest;

- Creep test, by applying constant shear stress and measuring the corresponding shear strain, at different shear stresses in the linear viscoelastic regime and above;

- Decreasing stress ramp to determine the flow curve, starting at a high stress corresponding to a shear rate of approximately $1000 \mathrm{~s}^{-1}$ or lower for the less concentrated sludge (to avoid turbulent conditions).

\section{Results and discussion}

Starting from rest, the shear stress sweep first elicits a linear viscoelastic response from the digested sludge up to a critical shear stress $\tau_{0}$ above which the material apparently starts to flow (Fig. 1). In the linear viscoelastic region, the behaviour is modelled by a generalised Kelvin-Voigt model, with a wide relaxation time spectrum modelled by a stretched exponential:

$$
\gamma(t)=\tau \cdot \frac{1}{G} \cdot\left(1-\exp \left(-(\lambda t)^{m}\right)\right)
$$

where $\gamma$ represents the strain, $\tau$ the stress and $\lambda=\frac{G}{\mu}$ with $G$ and $\mu$ the usual parameters of a

Kelvin-Voigt model.

Assuming that the sludge is flowing in its liquid regime above the critical shear stress following a Herschel-Bulkley model (Monteiro, 1997), the additional strain can be expressed as:

$$
\gamma(t)=\int_{t_{0}}^{t} \dot{\gamma} d u=\int_{t_{0}}^{t}\left(\frac{\tau-\tau_{0}}{K}\right)^{1 / n}=\int_{t_{0}}^{t}\left(\frac{a \cdot x-a \cdot t_{0}}{K}\right)^{1 / n} d x=\frac{a \cdot n}{(n+1) K}\left(t-t_{0}\right)^{1+1 / n}
$$


where $a$ is the slope of the shear stress ramp and $t_{0}$ the time such that the shear stress equals the yield stress of the Herschel-Bulkley model $\tau_{0}=a \cdot t_{0}$.

Thus, the total strain, which predicts the experimental data (Fig. 1), can be expressed as:

$$
\gamma(t)=\tau \cdot \frac{1}{G} \cdot\left(1-\exp \left(-(\lambda t)^{n}\right)\right)+b \cdot\left(t-t_{0}\right)^{1+1 / n}
$$

with $b=\frac{a n}{(n+1) \cdot K}$

Applying this to the experimental data gave a flow behaviour index for the Herschel-Bulkley model, $n$, greater than 1 (Fig. 1), meaning that the digested sludge could apparently be a shear-thickening liquid above $\tau_{0}$, which is unusual.

Creep tests confirmed a change in the behaviour above $\tau_{0}$. Below $\tau_{0}$, the strain slowly increased with time, while above $\tau_{0}$, the increase is faster (Fig. 2), both following a power law with time. However, even for stresses higher than $\tau_{0}$ (Fig. 2) the shear strain follows a power-law with a power-law index less than 1, indicating that the shear rate is a decreasing function of time: there is no steady state and so, sludge is restructuring and not flowing (otherwise, the shear rate would have been constant over time for a constant shear stress). The value $\tau_{0}$ cannot therefore be considered as a classical yield stress above which digested sludge flows in its liquid regime. These power-law relationships between strain and time are in fact a consequence of a structural relaxation process which occurs during creep (Baudez, 2008).

When the time of rest between the preshear and the stress sweep increases, the behaviour is globally the same, with first a linear viscoelastic behaviour (Fig. 3) but the critical shear stress, $\tau_{0}$ decreases with increase of the time of rest, the global elasticity decreases, the mean relaxation time (inverse of $\lambda$ ) increases and the strain corresponding to $\tau_{0}$ decreases (Fig. 4): the longer the time of rest, the smaller the linear viscoelastic range.

At rest, the digested sludge structure became weaker and weaker (decrease of $\tau_{0}$ and elasticity) but concurrently the relaxation time increased, indicating an evolution from a viscoelastic material towards a more elastic solid (the decrease of $\mu$ is faster than the decrease of $G$ ). Since this is physically impossible, this observed apparent behaviour is not representative of the true material behaviour but derives from erroneous interpretation of raw data.

Above $\tau_{0}$, experimental results showed the viscosity is globally decreasing, which is inconsistent with the apparent shear-thickening behaviour noted earlier, but oscillations of viscosity regarding shear stress are reported (Fig. 5). These oscillations indicated local minima in the flow curve where apparent shear rate occasionally decreased while shear stress increased.

If we assume the relationship between local shear rate and local shear stress is monotonic, then we can write:

$\dot{\gamma}=f(\tau)$ where $f$ is the inverse function of the behaviour law. 
In a Couette geometry, the shear rate can be expressed as:

150

$\dot{\gamma}_{\text {local }}=-r \cdot \frac{\partial(\omega)}{\partial r} \Leftrightarrow \omega=\int_{R_{1}}^{R_{2}} \frac{\dot{\gamma}_{\text {local }}}{r} d r \Leftrightarrow \omega=\int_{R_{1}}^{R} \frac{f\left(\tau_{\text {local }}\right)}{r} d r$

where $\omega$ is the angular velocity, $R_{1}$ the inner radius and $R-R_{1}$ the thickness of the sheared region. The maximum value of $R-R_{1}$ is $R_{2}-R_{1}$, where $R_{2}$ is the outer radius.

The apparent shear rate is calculated from the measured angular velocity, the only raw data measured by the rheometer. The shear rate given by the rheometer is calculated with the assumption of a full shear within the gap. So, if the apparent shear rate decreased, it means the angular velocity decreased. However, because $f$ is a monotonic function, this decrease of $\omega$ is rather the consequence of a decrease of the effective gap $R-R_{1}$, implying that shear banding has occurred during the measurement.

Such behaviour (shear banding and viscoelastic behaviour) has to be taken into account in digester design and operation, because shear banding means that there is coexistence of both sheared and unsheared zones in the digester, these last being useless, unmixed, dead zones.

According to Moller et al. (2008), the width of the flowing band can be directly related to the macroscopically imposed shear rate. At high shear rates, the whole gap is sheared and when the applied stress is much higher than $\tau_{0}$, the sludge flows normally, with no apparent perturbation effects, allowing us to have achieve reproducible measurements (Fig. 6) with the corresponding smooth classical shape of the flow curve.

As expected, the higher the concentration, the thicker the sludge (Fig. 7) but depending on the shear rate range, different well-known models can be used to describe the rheological behaviour of digested sludge. At high shear rates, a basic Bingham model is sufficient (Fig. 8) while at low and intermediate shear rates, Herschel-Bulkley and power-law models are more appropriate (Fig 9). They all represent the same material but can only be used in a specific range of validity, regarding the complexity of the process to be modelled. Thus, for pumping where shear rates are very high, a Bingham model would be appropriate since it deals with simple characteristics, i.e. a yield stress and a constant rheogram slope above it.

From a more general point of view, in the liquid regime we can summarize the rheological behaviour of digested sludge as a shear-thinning yield stress fluid with a plateau viscosity at high shear rates:

$\tau=\tau_{c}+\eta(\dot{\gamma}) \cdot \dot{\gamma}$ with $\eta(\dot{\gamma}) \underset{\dot{\gamma} \rightarrow \infty}{\longrightarrow} \alpha_{0}$

Moreover, at low and intermediate shear stresses, $\tau=\tau_{c}+K \cdot \dot{\gamma}^{n}=\tau_{c}+\eta(\dot{\gamma}) \cdot \dot{\gamma} \Leftrightarrow \eta(\dot{\gamma}) \approx K \cdot \dot{\gamma}^{n-1}$

Thus, for the sake of simplicity, we define the rheological behaviour of digested sludge as follows:

$$
\tau=\tau_{c}+\left(K \cdot \dot{\gamma}^{n-1}+\alpha_{0}\right) \cdot \dot{\gamma}
$$

On our range of data, i.e. below $1000 \mathrm{~s}^{-1}$, this model was successful. However, if $\dot{\gamma}<<1-n \sqrt{\frac{K}{\alpha_{0}}}$, the Herschel-Bulkley model is sufficient to model the behaviour, which corresponds to a shear rate 
188

189

190

191

smaller than $565 \mathrm{~s}^{-1}$ for the most concentrated sludge and smaller than $145 \mathrm{~s}^{-1}$ for the less concentrated sludge as shown below (Table 1).

Table 1: Shear rate above which the Herschel-Bulkley model is not suitable

\begin{tabular}{|c|c|}
\hline Concentration [\%] & Limit shear rate [s-1] \\
\hline 1.85 & 145 \\
\hline 2.56 & 280 \\
\hline 3.17 & 470 \\
\hline 4.89 & 565 \\
\hline
\end{tabular}

Equation (5) can also be expressed as:

$$
\begin{aligned}
& \tau=\tau_{c}+K \cdot \dot{\gamma}^{n}+\alpha_{0} \cdot \dot{\gamma} \\
& \Leftrightarrow \frac{\tau}{\tau_{c}}=1+\frac{K}{\tau_{c}} \cdot \dot{\gamma}^{n}+\frac{\alpha_{0}}{\tau_{c}} \cdot \dot{\gamma} \\
& \Leftrightarrow \frac{\tau}{\tau_{c}}=1+\beta \cdot \Gamma^{n}+\Gamma \\
& \Gamma=\frac{\alpha_{0}}{\tau_{c}} \cdot \dot{\gamma}, \beta=\frac{K}{\tau_{c}} \cdot\left(\frac{\tau_{c}}{\alpha_{0}}\right)^{n}
\end{aligned}
$$

In such a dimensionless form, all the flow curves are similar, independent of solids concentration (Fig. 10). From a physical point of view, this result means that there is some similarity of the network of interactions within the sludge at different concentrations, which is at the origin of the similarity of its macroscopic behaviour. In such suspensions, interactions can be classified into two main groups (Baudez and Coussot, 2001): hydrodynamic interactions (between solid particles and surrounding fluid, here basically represented by the Bingham viscosity) and non-hydrodynamic interactions (between solid particles, basically represented by the yield stress). Increasing the concentration doesn't change the nature of these interactions, but rather modifies their relative intensity. The dimensionless form smoothed these differences because both kinds of interactions in this form will approach unity.

On our range of concentrations, yield stress and Bingham viscosity increase with the solid concentration (Fig. 11) respectively following a power-law and an exponential law of the following form, which is in agreement with the literature, both for the yield stress (Baudez, 2008) and the Bingham viscosity (Sanin, 2002):

$\tau_{c}=\alpha \cdot\left(\phi-\phi_{0}\right)^{m}$

$k_{2}=\mu_{0} \cdot \exp (\beta \cdot \phi)$

where $\phi_{0}$ is the lowest concentration below which there is no yield stress, $m$ is related to the fractal dimension of sludge flocs (Baudez, 2008) and $\mu_{0}$ is the viscosity of the liquid medium. 
210 We found that the value $\mu_{0}$ is twice that of pure water, which can be explained by the large amount of dissolved matter present, which may increase the supernatant viscosity.

Conclusion

In this paper, we have shown that digested sludge is a shear-thinning yield stress fluid, presenting flow instabilities at low shear rates, manifesting as shear banding. At low shear stress, below the yield stress, digested sludge behaved as a viscoelastic solid. When the applied stress is increased, above a critical shear strain, which decreases with the restructuring, shear banding appears. Then, at higher stresses, digested sludge behaves like a yield stress fluid and can be modelled using both the Herschel-Bulkley and Bingham plastic models over a wide range of shear rates.

222 This behaviour was similar at different concentrations and yield stress followed a power-law with 223 the concentration while the Bingham viscosity followed an exponential law with concentration. By reducing the rheological parameters with the yield stress and the Bingham viscosity, which have to be measured separately, a master curve was obtained. This result means that the rheological behaviour of the digested sludge at any concentration can be deduced from this master curve.

However, further work has to be done on shear banding. This behaviour will have to be taken into account in digester design and process operations, in order to avoid dead zones in the digester.

Acknowledgements

The authors acknowledge the Cemagref-RMIT agreement for our collaboration

References

Appels, L., Baeyens, J., Degrève, J., Dewil, R., 2008. Principles and potential of the anaerobic digestion of waste-activated sludge. Progress in Energy and Combustion Science Volume 34, Issue 6, December 2008, Pages 755-781

Ayol, A., Filibeli, A; Dentel, S.K., 2006. Evaluaion of conditioning responses of thermophilicmesophilic anaerobically and mesophilic aerobically digested biosolids using rheological properties. Water Science and Technology, 54, 5, 23-31.

243 Baudez, J.C, Coussot, P., 2001. Rheology of aging, concentrated, polymeric suspensions 244 Application to pasty sewage sludges. J. Rheol. 45(5):1123-1139.

Baudez, J.C., 2006. About peak and loop in sludge rheogram. Journal of Environmental Management, 78, 232-239.

247 Baudez, J.C., 2008. Physical aging and thixotropy in sludge rheology. Applied Rheology, 18, $134951-8$. 
249 Forster, C.F., 1983. Bound water in sewage sludge and its relationship to sludge surfaces and 250 sludge viscosities. J. Chem. Tech. Biol., 33B:76-84.

251 Forster, C.F., 2002. The rheological and physico-chemical characteristics of sewage sludge". 252 Enzyme and Microb. Tech., 30(3):340-345.

253 Miller, E., Rothstein, J.P., 2007. Transient evolution of shear banding in wormlike micelle solutions. 254 J. Non-Newtonian Fluid Mech. 143, 22-37

255 Moller, P. C. F,Rodts, S. Michels, M. A. J. and Bonn,D., 2008. Shear banding and yield stress in 256 soft glassy materials. Phys. Rev. E 77, 041507.

257 Monteiro, P.S., 1997. The influence of the anaerobic digestion process on the sewage sludges 258 rheological behaviour. Wat. Sci. Tech., 36 (11): 61-67

259 Munoz, J., Alfaro, M.C., 2000. Rheological and phase behaviour of amphiphilic lipids. Grasas y 260 aceites, vol. 51, pp. 6-25

261 Namer, J. J. Ganczarczyk, L., 1993.Settling Properties of Digested Sludge Particle Aggregates. 262 Water Research, 27, 1285-1294

263 Sanin, F.D., 2002. Effect of solution physical chemistry on the rheological properties of activated 264 sludge. Water SA, 28: 207-212.

265 Slatter P. 1997. The rheological characterisation of sludges. Wat. Sci. Tech. 36(11), 9-18.

266 Slatter P. 2001. Sludge pipeline design, J. Wat. Sci. Tech. 44(10):115-120.

267 Slatter P. 2003. Pipeline transport of thickened sludges. Water 21: 56-57.

268 Tropey, W. N. and Melbinger, N. R., 1967. Reduction of Digested Sludge Volume by Controlled 269 Recirculation. Journal Water Pollution Control Federation 39(9): 1464-1474.

270 Sperry, W.A., 1959. Gas Recirculation at Aurora, Illinois, Sewage and Industrial Wastes vol. 31, n 2716. 


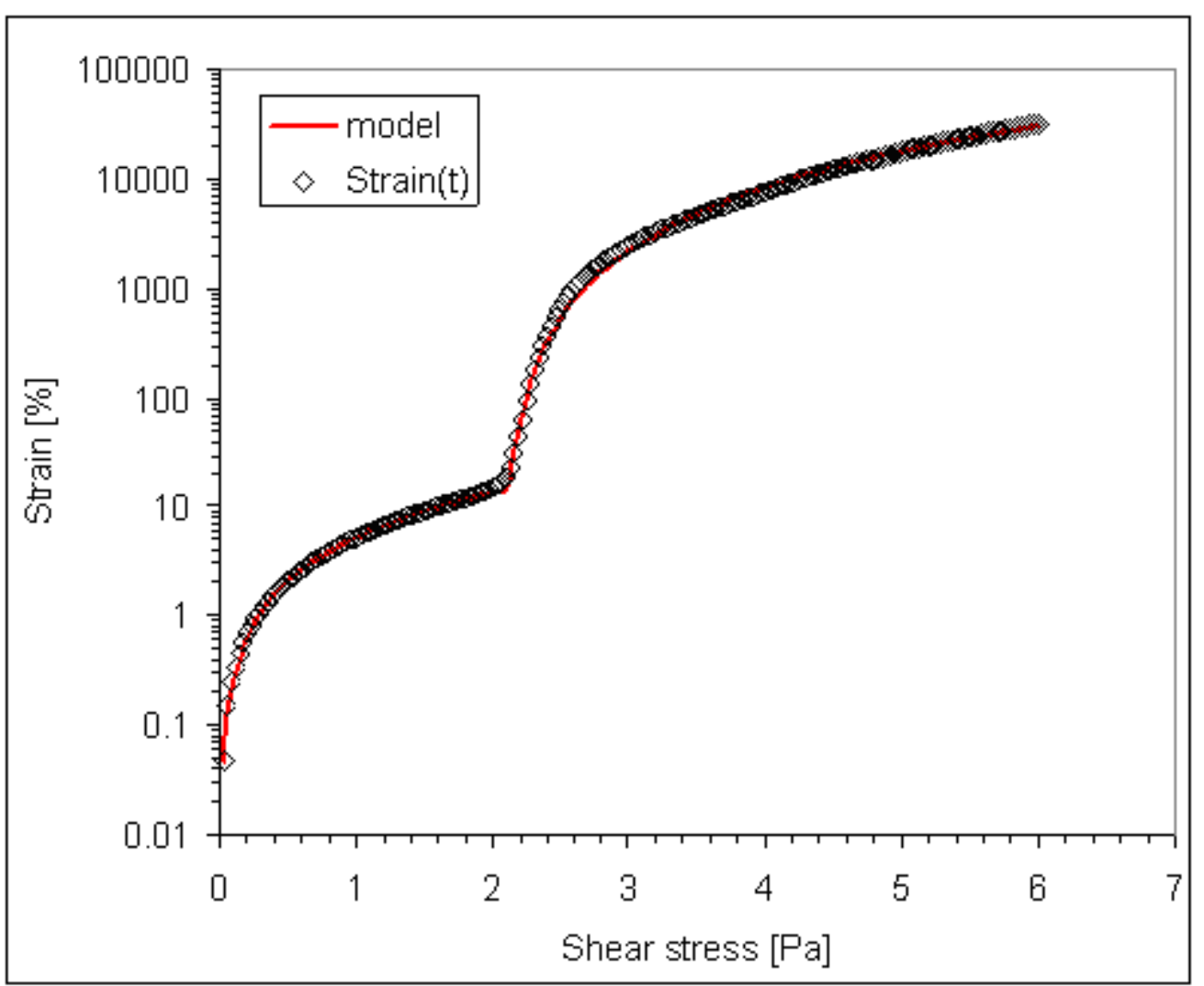

275 Figure 1: Strain-stress behaviour of the $4.9 \%$ digested sludge. The dashed lined corresponds to the 276 277 equals to $2.13 \mathrm{~Pa}$ and $\mathrm{n}=1.30$.

278

279 


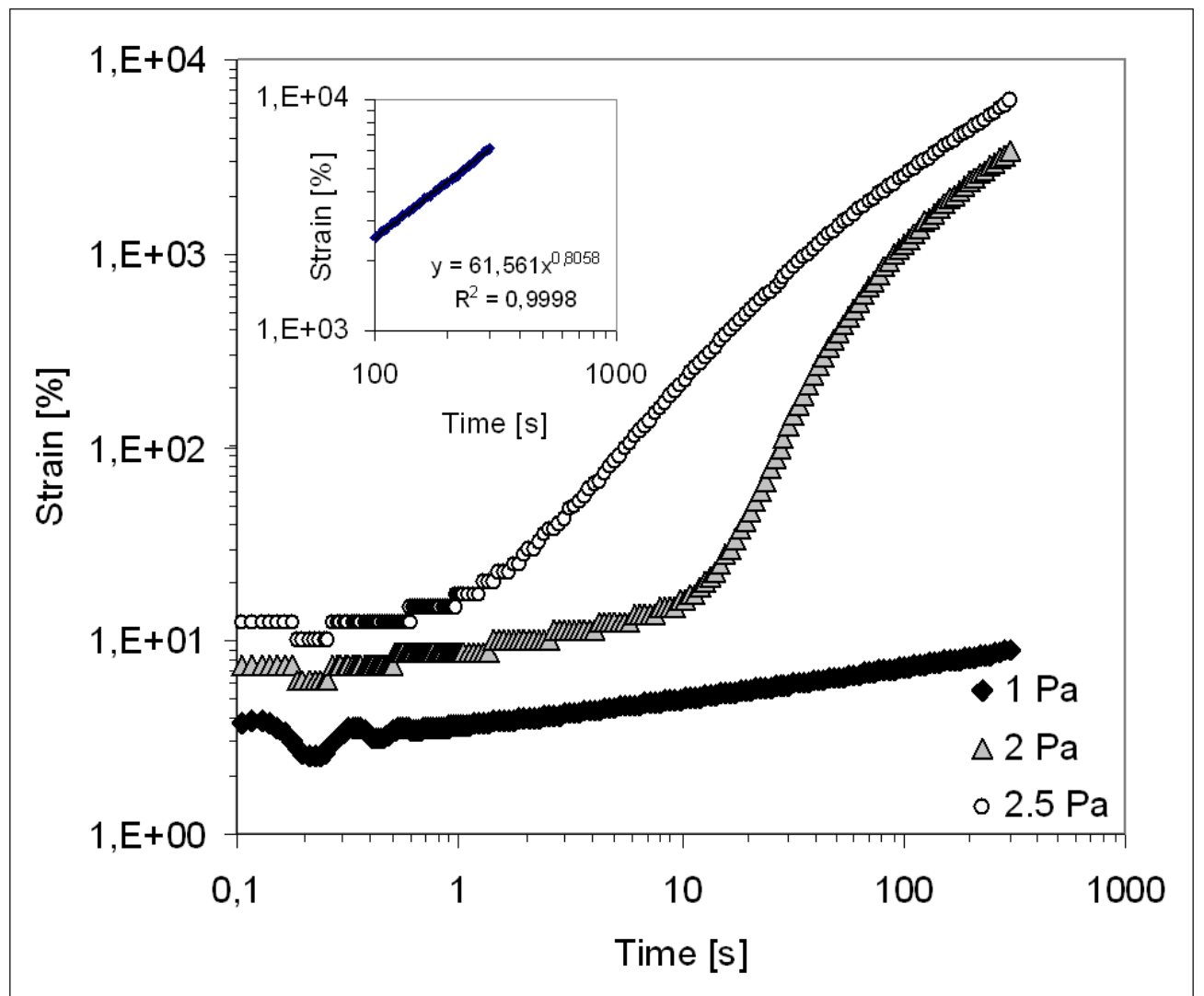

Figure 2: Creep test below, above and equal to the critical shear stress. Here, the critical stress is 2.5

$\mathrm{Pa}$ for the 4,9\% sludge. The insert is a focus on the strain at the highest strain at longer time, following a power-law with an index smaller than 1.

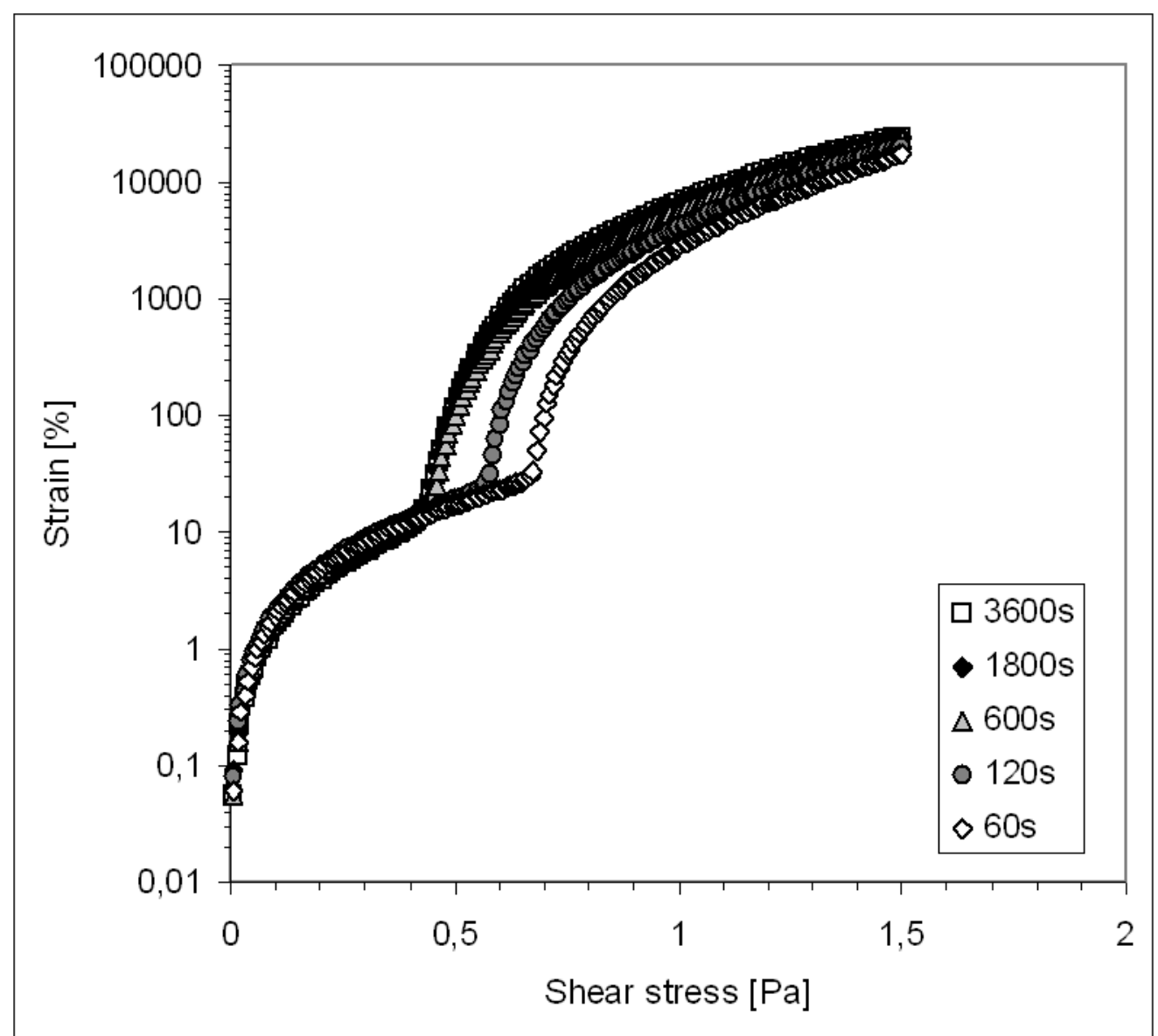

Figure 3: strain stress behaviour when a stress sweep is applied after different time of rest. 


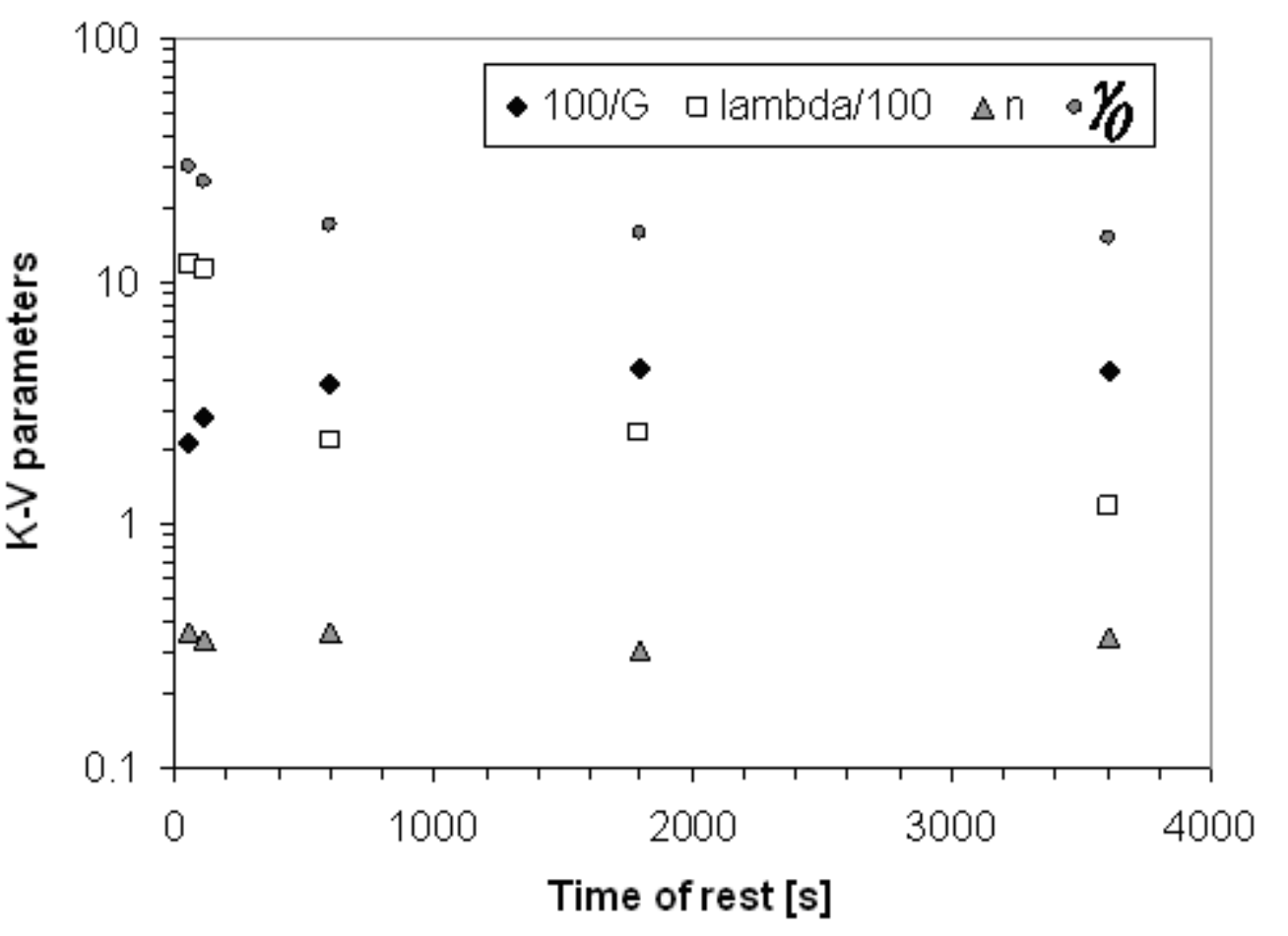

Figure 4: Evolution of the Kelvin-Voigt model parameters as a function of the time of rest.

293

294

295

296

297 


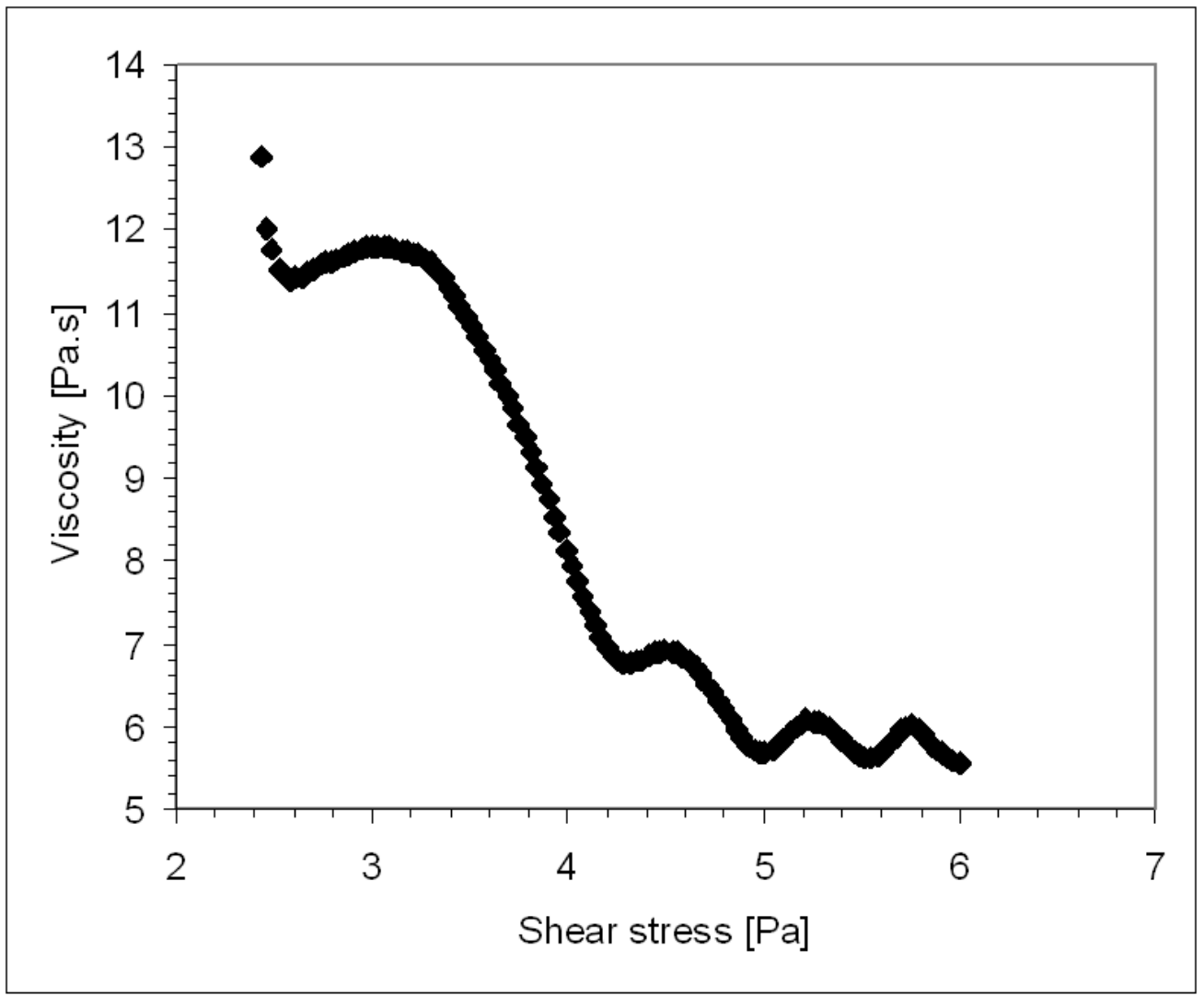

Figure 5: stress-viscosity variations highlighted oscillations with the $4.9 \%$ digested sludge. 300

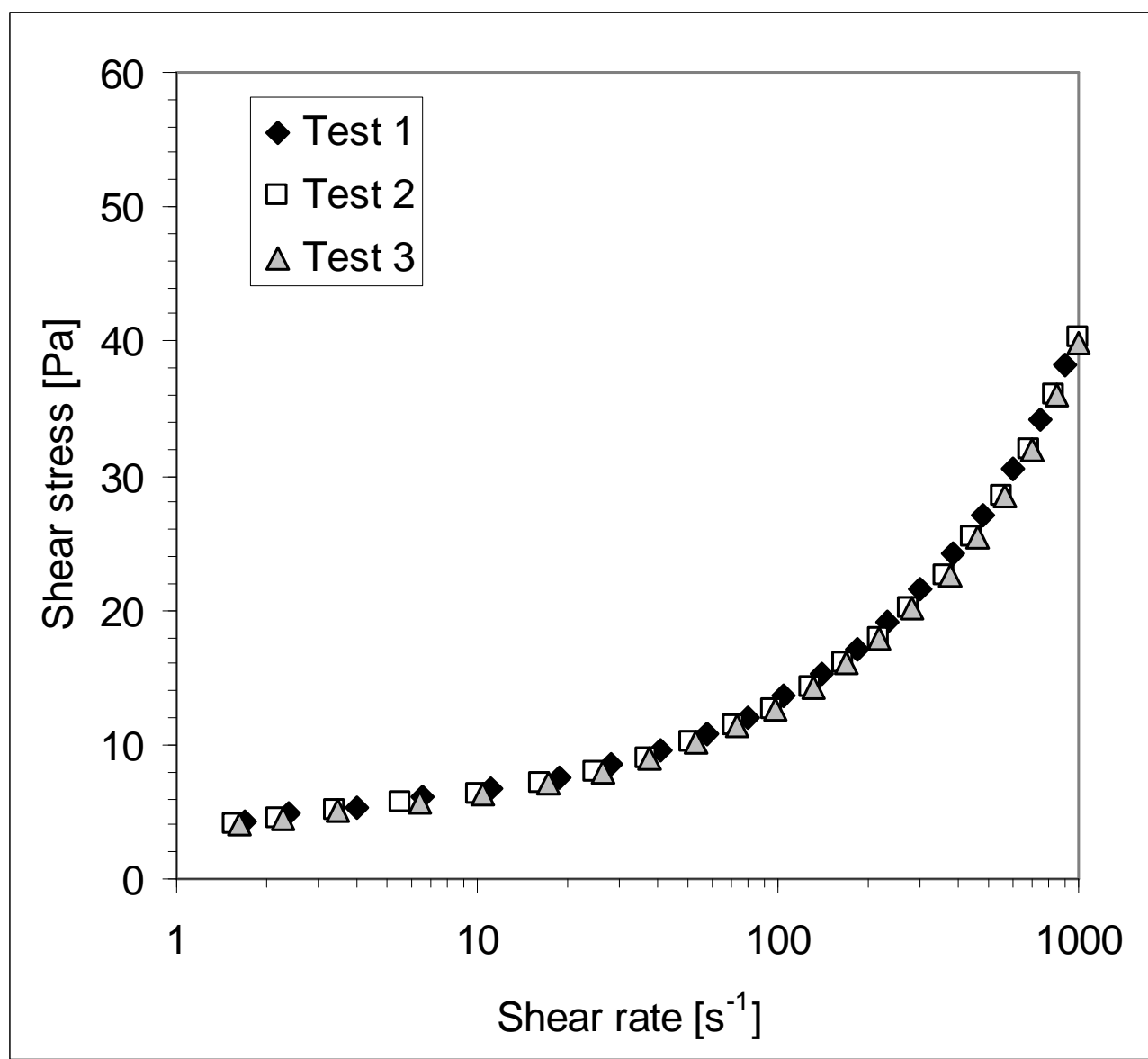

Figure 6: Repeatability of the measurements. 


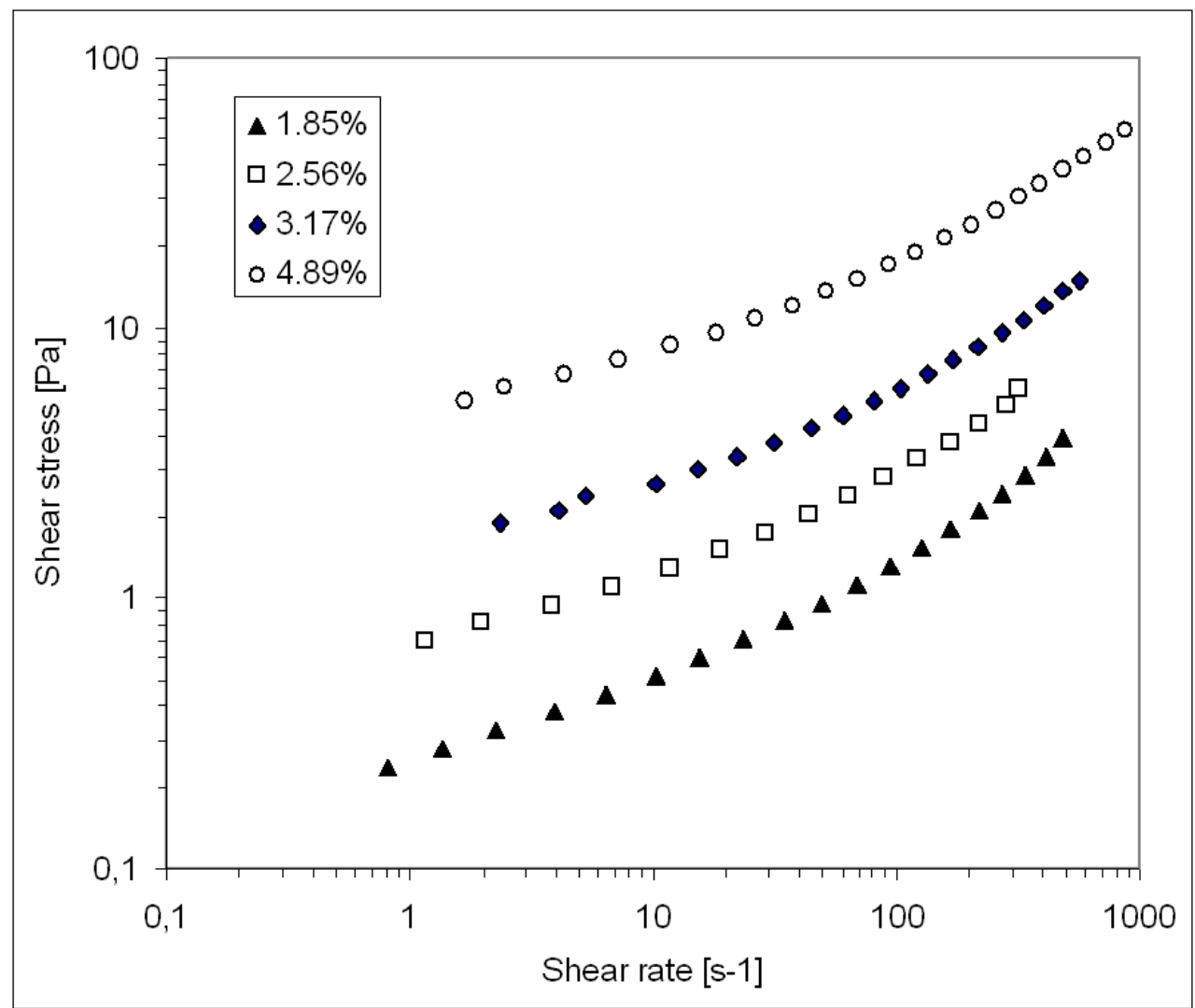

Figure 7: Flow curves regarding the concentration of the digested sludge.

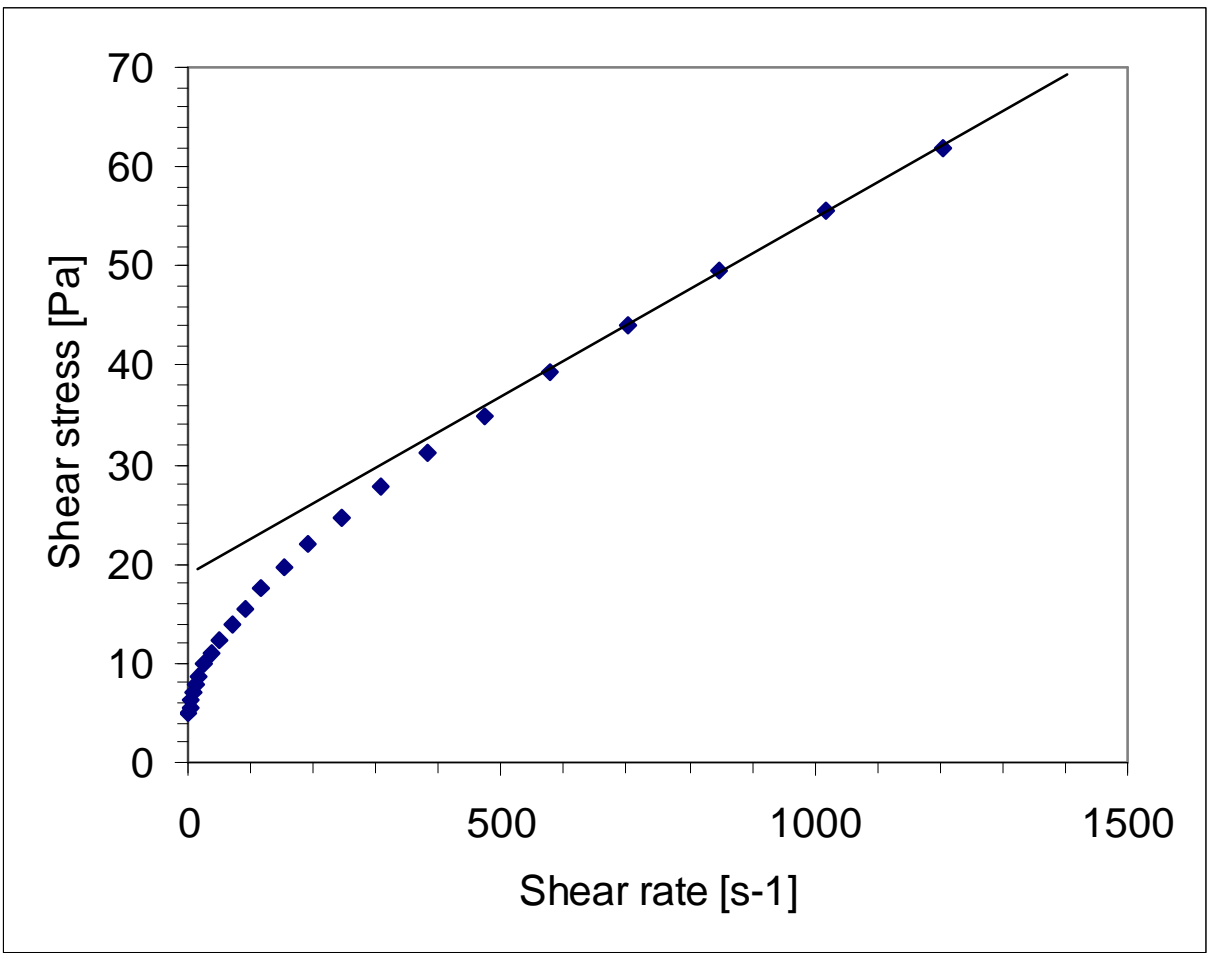

Figure 8: At high shear rates, the rheological behaviour can be basically modelled with a Bingham plastic model. 
313

314

315 316

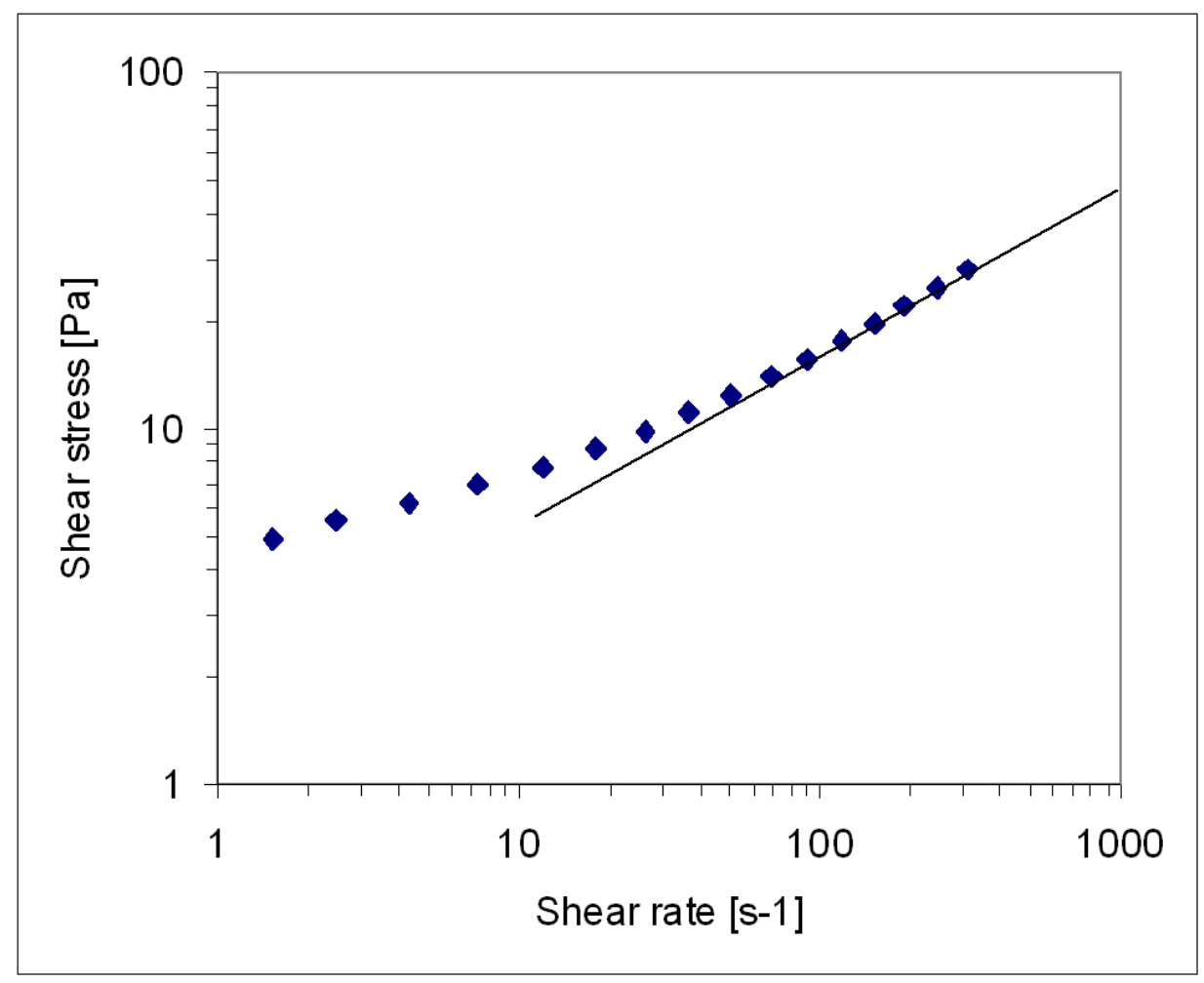

Figure 9: At low and intermediate shear rates, the Herschel-Bulkley model or power-law model are the most suitable. The dashed line represents the power-law model $\tau=2.05 \cdot \dot{\gamma}^{0.45}$.

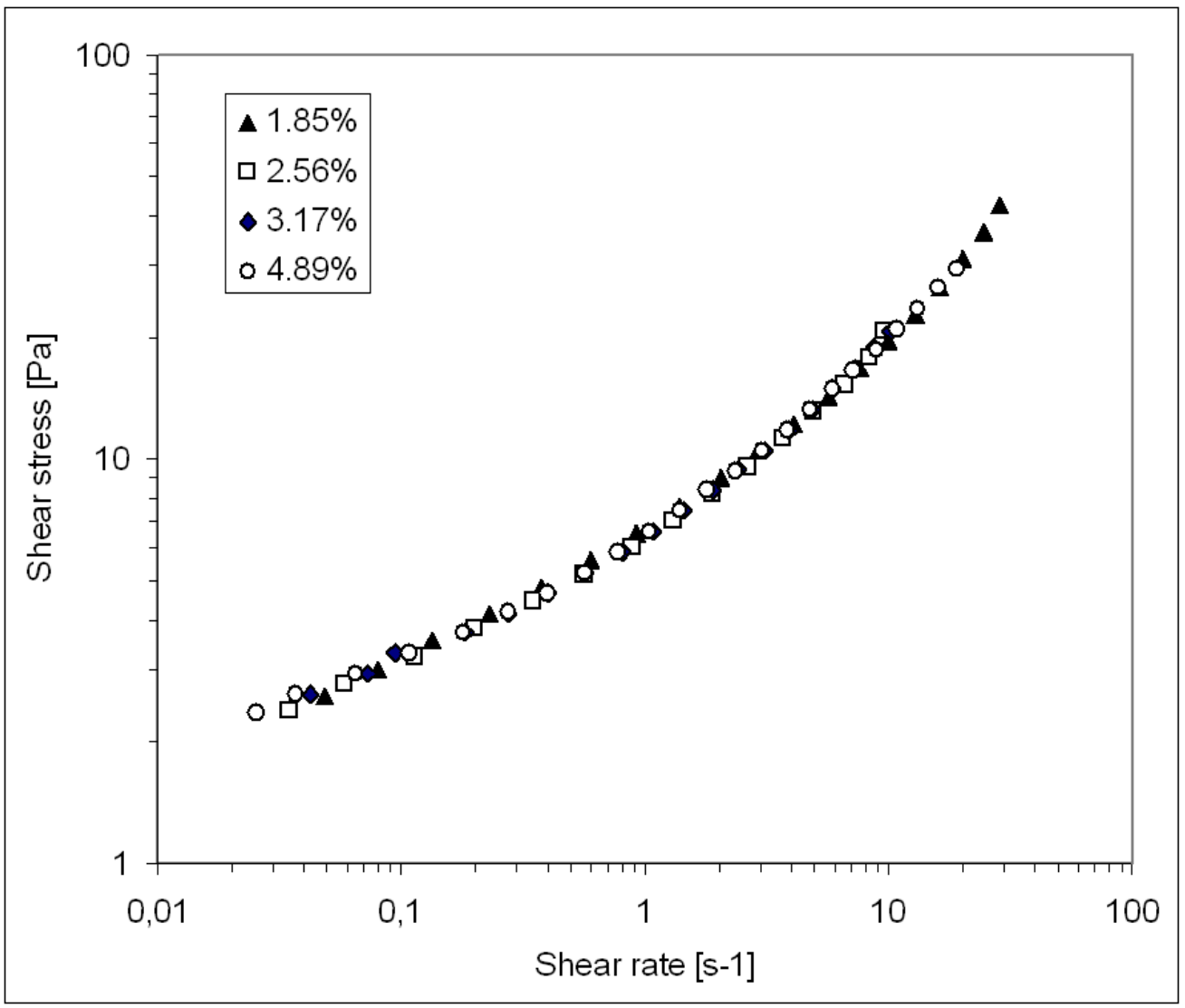

Figure 10: Dimensionless flow curves of the digested sludge at different concentrations. 


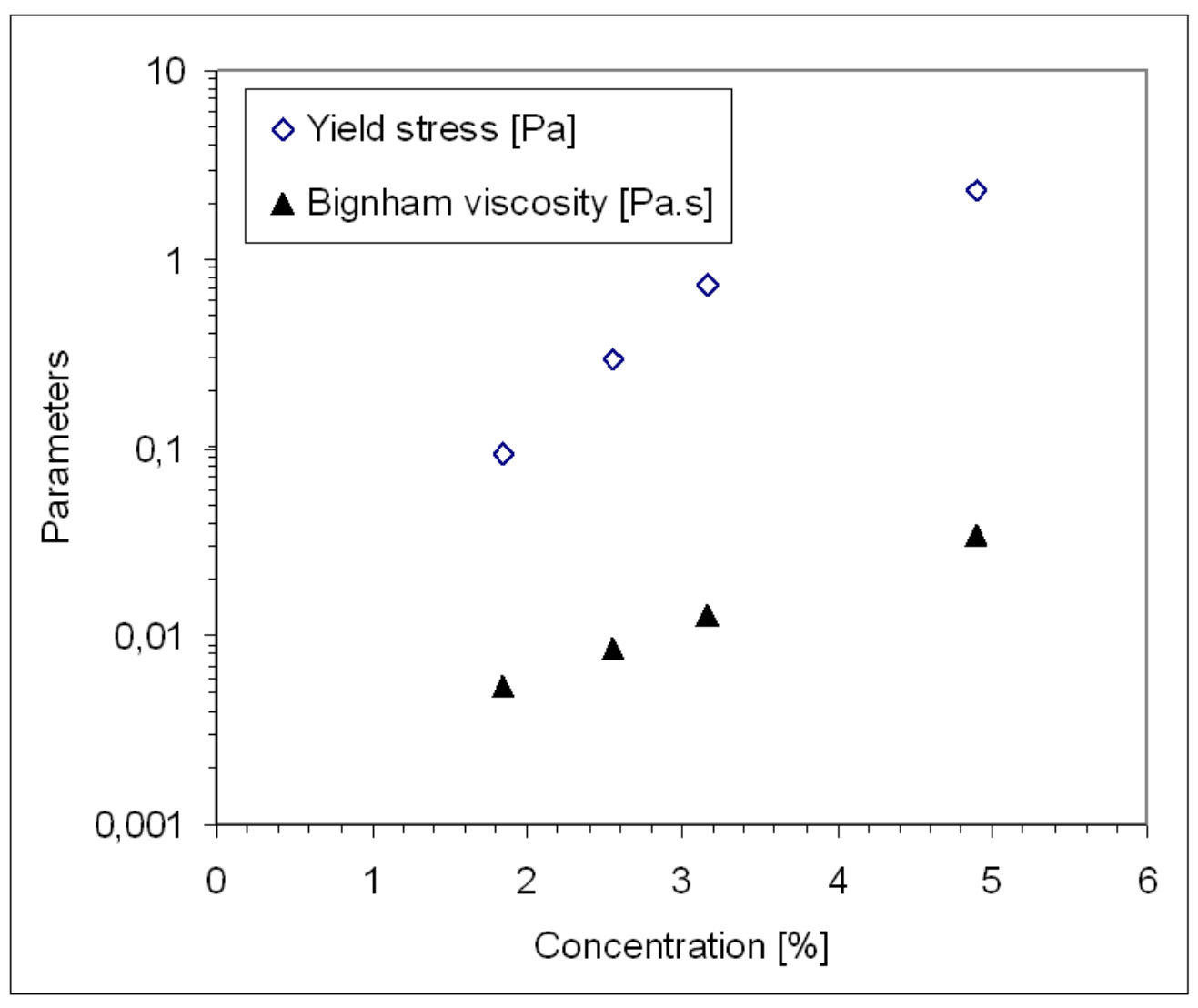

321 Figure 11: Evolution of the yield stress and the Bingham viscosity regarding the concentration. The 322 parameters of the equations (7) and (8) are respectively $a=0.19 P a, \phi_{0}=1.17 \%, m=1.89$ and 323 $\mu_{0}=0.0018$ Pa.s, $\beta=0.604$. 